(C) 2010 IEEE. Personal use of this material is permitted. Permission from IEEE must be obtained for all other uses, in any current or future media, including reprinting/republishing this material for advertising or promotional purposes, creating new collective works, for resale or redistribution to servers or lists, or reuse of any copyrighted component of this work in other works. 


\title{
A SPECT Scanner for Rodent Imaging Based on Small-Area Gamma Cameras
}

\author{
Eduardo Lage, José L. Villena, Gustavo Tapias, Naira P. Martínez, Maria L. Soto-Montenegro, \\ Mónica Abella, Alejandro Sisniega, Francisco Pino, Domènec Ros, Javier Pavía, Manuel Desco, and \\ Juan J. Vaquero, Senior Member, IEEE
}

\begin{abstract}
We developed a cost-effective SPECT scanner prototype (rSPECT) for in vivo imaging of rodents based on small-area gamma cameras. Each detector consists of a position-sensitive photomultiplier tube $(\mathrm{PS}-\mathrm{PMT})$ coupled to a $30 \times 30 \mathrm{NaI}(\mathrm{TI})$ scintillator array and electronics attached to the PS-PMT sockets for adapting the detector signals to an in-house developed data acquisition system. The detector components are enclosed in a leadshielded case with a receptacle to insert the collimators. System performance was assessed using ${ }^{99 \mathrm{~m}} \mathrm{Tc}$ for a high-resolution parallel-hole collimator, and for a $0.75-\mathrm{mm}$ pinhole collimator with a $60^{\circ}$ aperture angle and a $42-\mathrm{mm}$ collimator length. The energy resolution is about $10.7 \%$ of the photopeak energy. The overall system sensitivity is about $3 \mathrm{cps} / \mu \mathrm{Ci} /$ detector and planar spatial resolution ranges from $2.4 \mathrm{~mm}$ at $1 \mathrm{~cm}$ source-to-collimator distance to $4.1 \mathrm{~mm}$ at $4.5 \mathrm{~cm}$ with parallel-hole collimators. With pinhole collimators planar spatial resolution ranges from $1.2 \mathrm{~mm}$ at $1 \mathrm{~cm}$ source-to-collimator distance to $2.4 \mathrm{~mm}$ at $4.5 \mathrm{~cm}$; sensitivity at these distances ranges from 2.8 to $0.5 \mathrm{cps} / \mu \mathrm{Ci} /$ detector. Tomographic hot-rod phantom images are presented together with images of bone, myocardium and brain of living rodents to demonstrate the feasibility of preclinical small-animal studies with the rSPECT.
\end{abstract}

Index Terms-Single Photon Emission Computed Tomography (SPECT), small-animal imaging, small-area gamma camera.

Manuscript received January 05, 2010; revised April 16, 2010; accepted June 25, 2010. Date of publication September 13, 2010; date of current version October 15, 2010. This work was supported in part by the CD-TEAM project, CENIT program, Spanish Ministerio de Industria and with grants from the Ministerio de Educación y Ciencia, Projects TEC2007-64731/TCM, TEC2008-06715-C02-01, SAF2009-08076, program ARTEMIS S2009/DPI-1802, Comunidad de Madrid, and the RECAVA-RETIC Network.

E. Lage, J. L. Villena, G. Tapias, M. Abella, and A. Sisniega are with Unidad de Medicina y Cirugía Experimental, Hospital General Universitario Gregorio Marañon, Madrid, Spain (e-mail: elage@ mce.hggm.es; josvilo@yahoo.es; gustavo.tapias@gmail.com; mabella@mce.hggm.es; asisniega@mce.hggm.es).

N. P. Martínez and F. Pino are with Unidad de Biofísica y Bioingeniería, Universidad de Barcelona-IDIBAPS, Barcelona, Spain (e-mail: nairamv@gmail. com; fpino@medicina.ub.es).

M. L. Soto-Montenegro is with Unidad de Medicina y Cirugía Experimental, Hospital General Universitario Gregorio Marañon, Madrid, Spain and also with CIBERSAM, Madrid, Spain (e-mail: marisa@mce.hggm.es).

D. Ros is with Unidad de Biofísica y Bioingeniería, Universidad de Barcelona- IDIBAPS, Barcelona, Spain and also with CIBER-BBN, Barcelona, Spain (e-mail: domenec@ub.es).

J. Pavía is with Servicio de Medicina Nuclear, Hospital Clínico y Provincial de Barcelona, Barcelona, Spain and also with CIBER-BBN, Barcelona, Spain (e-mail: jpavia@clinic.ub.es).

M. Desco is with Unidad de Medicina y Cirugía Experimental, Hospital General Universitario Gregorio Marañon, Madrid, Spain, CIBERSAM, Madrid, Spain, and also with the Departmento de Bioingeniería e Ingeniería Aeroespacial, Universidad Carlos III de Madrid, Spain (e-mail: desco@mce.hggm.es).

J. J. Vaquero is with the Departmento de Bioingeniería e Ingeniería Aeroespacial, Universidad Carlos III de Madrid, Spain (e-mail: juanjose.vaquero@uc3m. es).

\section{INTRODUCTION}

0 INCE the application of the pinhole imaging principle to SPECT, several scanners dedicated to rodents have been developed, thus providing an alternative technique to ex-vivo measurement of biological parameters. The earliest small-animal SPECT systems were configured with one to four clinical scintillation cameras operated using a collimator with a single pinhole aperture [1]-[3]. These systems allow high spatial resolution and small field of view projection data to be obtained by using high magnification factors and moderate spatial resolution detectors. However, this approach requires a heavy collimated detector to rotate around a small object with a constant radius of rotation (thus challenging the mechanical implementation) or to rotate the animal (thus making difficult its use with living samples). Currently, the trade-off between spatial resolution and detection sensitivity in most small-animal systems (based on either clinical gamma cameras or custom detectors) has improved by an effective use of the active area with multipinhole collimators, thus increasing acquisition speed and optimizing field of view size [4]-[10]. Nevertheless, as the number of pinholes increases, the detector area required for the resulting projections also increases with the consequent rise in material cost. Although these newer systems provide a wide range of possibilities for translational research, most common protocols encountered in practice can also be accomplished using more cost-effective solutions with lower performance. These include small-animal tomographic systems [11]-[15] and planar imaging systems [16]-[19] based on small-area gamma cameras. This type of solution based on compact gamma cameras is relatively inexpensive and has also been extensively investigated for clinical purposes [20]-[22].

In this work we present the rSPECT, a prototype scanner for in vivo imaging of rodents implemented to evaluate the feasibility of constructing a 4-head SPECT/CT commercial system with coplanar configuration (similar to that reported in [23]). This prototype is based on two small-area $(44.4 \mathrm{~mm} \times 44.4 \mathrm{~mm}$ active area per camera) and high-resolution gamma cameras mounted opposite each other on a robotic gantry. The spatial resolution and sensitivity can be adjusted depending on the study requirements by interchanging the collimators (parallel-hole or pinhole with different apertures) and adjusting the radius of rotation (ROR). To compensate for the intrinsic lack of magnification derived from small-area detectors and the relatively poor resolution of parallel-hole collimators, iterative reconstruction algorithms with accurate system modeling were used. Performance of the detectors was evaluated in terms of energy resolution and integral uniformity without collimators, and in terms of 
spatial resolution and sensitivity using the parallel-hole collimators and the pinhole collimators. Tomographic hot-rod phantom images are presented together with images of bone, myocardium and brain of living rodents to demonstrate the feasibility of preclinical small-animal studies with the rSPECT.

\section{Materials AND Methods}

\section{A. Gamma Cameras}

Each gamma camera was built using a Hamamatsu H-8500 position-sensitive photomultiplier tube (PS-PMT) coupled to a $\mathrm{NaI}(\mathrm{Tl})$ scintillator array through a thin layer of optical grease (BC-630, Bicron-St. Gobain, Paris, France). The scintillator matrices were also assembled by Bicron-St. Gobain and consist of $30 \times 30$ pixels of $1.4 \times 1.4 \times 6 \mathrm{~mm}^{3}$. The crystals are separated by a $200-\mu \mathrm{m}$ thick white reflector material which also covers its front side. The array is viewed through a $3 \mathrm{~mm}$ glass window and encapsulation is completed by a $50-\mu \mathrm{m}$ thick aluminum cover. The photomultiplier have 12 stages of metal channel dynode and $8 \times 8$ multiple anodes, providing an active area of roughly $49 \mathrm{~mm} \times 49 \mathrm{~mm}$. Each detector includes a compact PCB (Printed Circuit Board) stack directly attached to the PS-PMT sockets, containing a symmetric charge division circuit [24], [25]. Using this scheme the 64 anode signals of the PS-PMT are read out via a matrix of 128 resistors (one " $X$ " and one "Y" per pad) arranged to allow the $\mathrm{X}$ and the $\mathrm{Y}$ signals from one tube to be represented by 8 signal lines for each dimension (8 X-channel x 8 Y-channel). The PCB stack also includes a high-voltage supply and amplification stages for the resulting position signals and for a timing signal from the PS-PMT [26]. The scintillator array, the PS-PMT, and the electronics are assembled in a black-delrin enclosure which fits in a lead-covered case (Fig. 1(a)). This enclosure contains a mechanism that allows easy changing of the collimator depending on the study requirements. The camera configurations evaluated in this work are based on a) a low-energy and high-resolution parallel-hole collimator (1.2-mm hexagonal holes, 20-mm thick, and 0.2-mm septal thickness) primarily for (whole body) rat studies and planar imaging, and b) a low-energy pinhole collimator designed for organ-focused and (whole body) mice imaging, with a 42-mm collimator length, 8-mm-thick lead shielding, a $60^{\circ}$ aperture angle, and interchangeable tungsten inserts (knife-edge type) with different aperture sizes (Fig. 1(b)).

\section{B. Acquisition Electronics}

The data acquisition system of the rSPECT (Fig. 2) consists of an arbiter module implemented using a programmable logic device (PLD) which controls the generation and distribution of trigger signals, two 16-channel ADC cards (12-bit resolution) for data digitization, and a 32-bit digital I/O PCI card (NI-6533DIO-32HS, National Instruments Corporation) for data transmission to a computer.

Each ADC card is used to simultaneously digitize the 8-X and 8-Y position signals of a single detector after the detection of a valid event. An additional PLD is located in each of these modules for handling digitization and data-transfer to the acquisition computer. Additionally, eight general purpose 12-bit counters

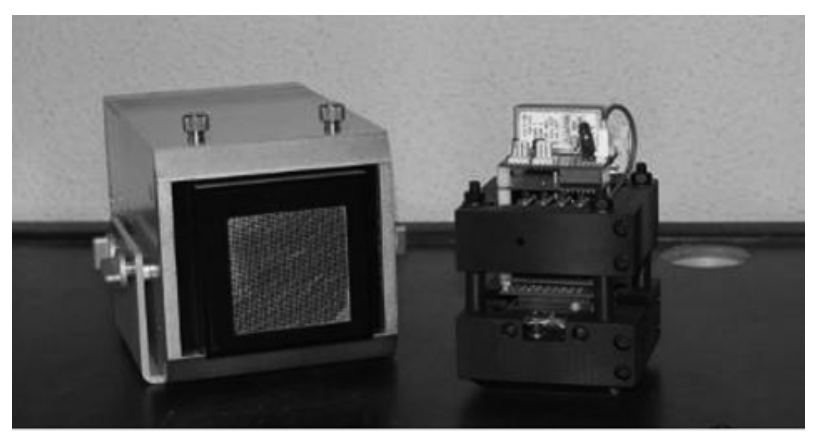

(a)

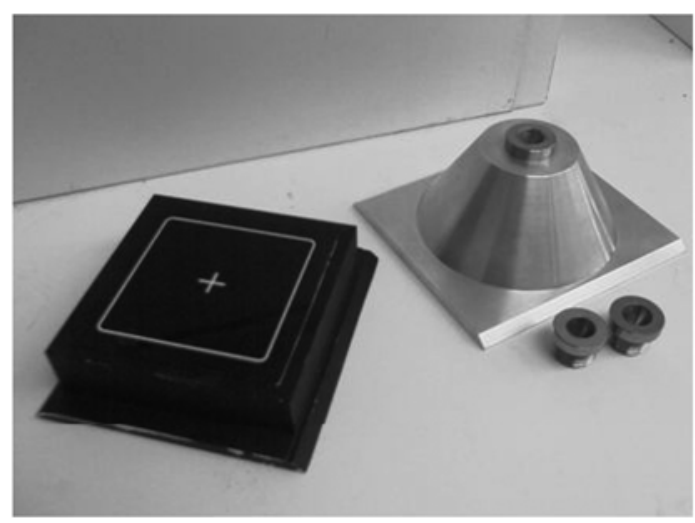

(b)

Fig. 1. (a) Lead-shielded case equipped with a parallel-hole collimator (left) and SPECT detector (right); (b) parallel-hole collimator (left) and pinhole collimator with different apertures (right).

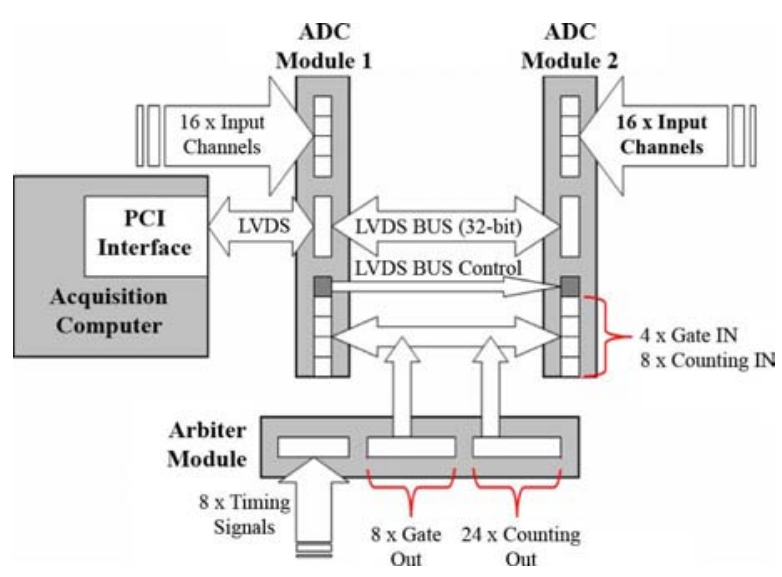

Fig. 2. rSPECT data acquisition system components and topology. The system contains an arbiter module, two ADC modules and a digital I/O PCI interface. The ADC modules and the PCI card are connected using a 32-bit LVDS bus.

per ADC card were located inside the PLD (count input signals are accessible in ADC sockets, see Fig. 2). The ADC cards and the digital I/O PCI interface are connected using a high-speed 32-bit LVDS (Low Voltage Differential Signaling) bus. In this architecture, ADC modules are also daisy-chained in order to access the LVDS bus coherently. Each ADC channel and data counter also includes a 4-bit header to identify data frames.

The timing signals of the detectors are fed into pulse discriminator circuits located in the arbiter module. These circuits consist of a pair of discriminators per detector (lower- and upperlevel), configured to detect photons with energy within the range 


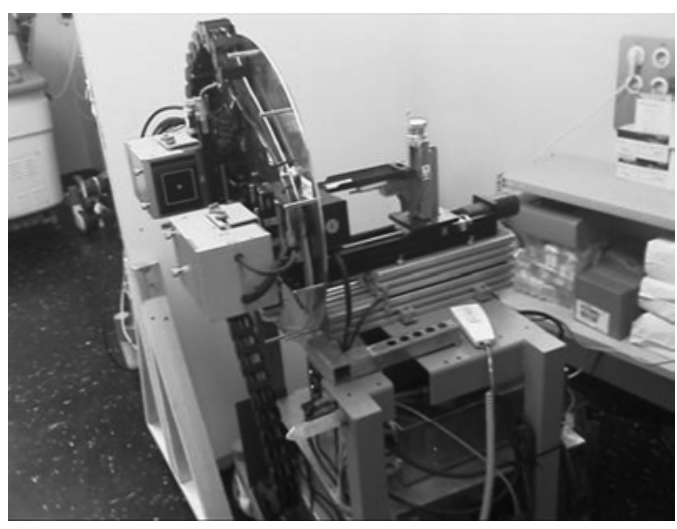

Fig. 3. rSPECT system prototype. The cameras are mounted on a robotic gantry containing six motorized movement axes. The entire system is controlled by a single PC used to synchronize data capture with mechanical movements during acquisitions.

defined for the discriminator thresholds. For ${ }^{99 \mathrm{~m}} \mathrm{Tc}$-based acquisitions these thresholds were set respectively to $10 \mathrm{keV}$ and $180 \mathrm{keV}$. After the detection of a valid event, trigger signals are generated and transmitted to the corresponding ADC module/s to start the digitization of the position signals necessary to characterize the event. These position signals are integrated for a 900-ns period, digitized and sent to the PC for processing. The current value of the counters included in each ADC card is sent together with the data frame for each event acquired. The data contained in these counters provides synchronization information such as the detector position (by a reference signal generated from an encoder located in the rotatory gantry), global time (from a 500-KHz clock signal from the arbiter module) or gating signals (from an external module).

\section{C. rSPECT Prototype}

The small-animal SPECT prototype is based on two gamma cameras mounted opposite each other on a motorized gantry (Fig. 3). The mechanical system consists of six movement axes controlled by a computer with RS-232 communication and a set of digital drives (ISCM-4805, Technosoft) interconnected using a CAN bus. Four motorized axes are mounted on the rotary stage of the gantry thus allowing detectors to be displaced in the tangential and radial directions for the adjustment of radius and center of rotation. A sample holder enables the sample to be moved along the axial direction. The entire system is controlled by a single PC running a Linux operating system. Control software, written in ANSI C, is used to synchronize data capture with mechanical movements in order to implement the different acquisition protocols. During scans, this software receives data from the PCI interface and processes them to correct pedestal values of $\mathrm{ADC}$ channels, to calculate the position of interaction (using a center of gravity algorithm with the 8-X and 8-Y position signals) and energy deposited by the photons (obtained as the sum of these position signals), and to generate list-mode files containing this information and synchronization events from the counters.

Scan planning, data processing, image reconstruction, and image display are managed from a remote computer running an
IDL-based (ITT Visual Information Solutions) console which also provides user interface and analysis tools [27].

\section{Calibration and Data Processing}

After acquisition, the list-mode files are processed by the user interface and converted into corrected projections. This process consists of a first step including the detector-related corrections such as camera non-uniformity, non-linearity or energy peaking, and a second step in which issues associated with detector misalignments are corrected.

For the detector-related corrections, a ${ }^{99 \mathrm{~m}} \mathrm{Tc}$ point source $(1.3 \mathrm{mCi})$ located at a distance of $25 \mathrm{~cm}$ from the front face of the cameras was acquired without collimators ( $>10,000$ counts per pixel). A second acquisition of similar characteristics but using ${ }^{18} \mathrm{~F}$ instead of ${ }^{99 \mathrm{~m}} \mathrm{Tc}$ was also carried out without collimators. We used the flood-field images obtained with ${ }^{99 \mathrm{~m}} \mathrm{Tc}$ $(256 \times 256$ pixels) to create a lookup table (LUT) to associate each scintillator pixel $(30 \times 30$ crystals $)$ with a region on the PS-PMT. Based on that table, the pulse-height spectrum is generated for each crystal using the data acquired with ${ }^{18} \mathrm{~F}$ and ${ }^{99 \mathrm{~m}} \mathrm{Tc}$, and the position of the photopeaks are used to calculate the correspondence between ADC channels and keV. The energy-calibration factors obtained in this way are saved together with the photopeak channels of each crystal and used to set the energy window of acquisitions. Next, we compared the number of counts recorded within each crystal with the mean pixel value of the image acquired with ${ }^{99 \mathrm{~m}} \mathrm{Tc}$, thus obtaining a non-uniformity correction file with the relative efficiency of each image element. Each subsequently acquired image is processed using the corresponding crystal LUT and the energy and uniformity correction data.

The detectors and center of rotation (COR) misalignments are calculated by a second calibration protocol performed with pinhole collimators using a method similar to that described in [3]. After estimation of correction factors, misalignments in radial and tangential planes were mechanically corrected using the linear stages included on the gantry. After this initial calibration, which avoids large misalignments, further corrections were performed. Standard software correction of the center of rotation was carried out for the parallel-hole collimators. Regarding the pinhole collimator, the SPECT system was calibrated using the method proposed in [28].

Uniformity, energy, and alignment corrections proved to be very stable (more than three months) if the machine is not turned off and the room temperature remains within reasonable limits $\left(<35^{\circ} \mathrm{C}\right)$.

\section{E. Pinhole and Parallel-Hole SPECT Reconstruction}

Analytical and statistical algorithms were used for image reconstruction. With regards to analytical methods we applied a standard filtered backprojection algorithm for parallel-hole based acquisitions, and the FDK algorithm [29] for pinhole based acquisitions. The statistical techniques used were based on an iterative ordered subsets-expectation maximization (OSEM) algorithm [30]. The algorithms applied for the parallel collimator were adapted to the small-animal SPECT scanner from 2D [31] and 3D [32] algorithms previously dȩveloped 
for clinical cameras. The algorithm for the pinhole collimator was also an in-house version of [32] adapted to this specific geometry. In this case, to avoid axial artifacts, the reconstruction was limited to those axial slices where each voxel in the field-of-view contributes to all projections. Moreover, the displacement of the bed between two successive acquisitions (whole body studies) was less than the size of the reconstructed volume in z-direction. All reconstruction iterative algorithms included compensation of the spatially varying collimator response. However, for pinhole collimators, only geometric collimator response was considered. Pinhole penetration and the depth-of-interaction in the detector array as a function of the angle-of-incidence on the detectors were not modeled.

\section{F. Performance Evaluation}

Planar measurements were carried out for both cameras and the different available collimators (high-resolution parallel-hole collimators and pinhole collimators equipped with $0.75-\mathrm{mm}$ apertures). To determine planar spatial resolution of the system, a single $0.3-\mathrm{mm}$ inner diameter glass capillary source filled with $2.1 \mathrm{mCi}$ of ${ }^{99 \mathrm{~m}} \mathrm{Tc}$ was used. Before acquisition, the line source and the cameras were leveled axially in such a way that the image was projected onto the central pixels of both detectors. On the first measurement, the source-to-collimator distance was $10 \mathrm{~mm}$ with both collimator types. Cameras were stepped in 5-mm increments until a 45-mm source-to-collimator distance was reached. Projections were acquired for 120 seconds at each camera position using a $20 \%$ symmetric energy window. FWHM spatial resolution of each projection was determined by fitting a Gaussian function to the count profile of each row of crystals and averaging them.

The integral uniformity of each camera was measured using the field-flood images obtained with ${ }^{99 \mathrm{~m}} \mathrm{Tc}$ over the useful (UFOV, $28 \times 28$ crystals) and central (CFOV, $21 \times 21$ crystals) fields of view. The energy performance was evaluated at 140 $\mathrm{keV}$ for each usable pixel by fitting the channels near the photopeak to a Gaussian function. The energy resolution was then calculated as the FWHM of the Gaussian function divided by the photopeak energy in percentage.

Finally, a square plastic phantom $(5 \mathrm{~mm} \times 5 \mathrm{~mm} \times 8 \mathrm{~mm})$ filled with an aqueous solution of ${ }^{99 \mathrm{~m}} \mathrm{Tc}(2.6 \mathrm{mCi})$ was used to evaluate camera sensitivity at different source-to-collimator distances. The initial distance (measured from the center of the phantom) was $15 \mathrm{~mm}$ with both collimator types. Cameras were stepped in 5-mm increments until a 45-mm distance was reached. Projections were acquired for $120 \mathrm{~s}$ at each position using a $20 \%$ symmetric energy window.

\section{G. SPECT Imaging}

The spatial resolution was evaluated in tomographic images using a micro-Derenzo hot-rods phantom (Ultra-Micro Hot Spot Phantom, Data Spectrum Corporation, Hillsborough). This phantom has six sectors with rod diameters of 2.4, 2.0, $1.7,1.35,1.0$, and $0.75 \mathrm{~mm}$. The phantom was filled with 4.5 $\mathrm{mCi}$ of ${ }^{99 \mathrm{~m}} \mathrm{Tc}$ and acquired for two hours using the pinhole collimators equipped with the $0.75-\mathrm{mm}$ aperture. A total of 120 projections were acquired over $360^{\circ}$ with a $33.5-\mathrm{mm}$ radius of rotation $(\mathrm{ROR})$.
In order to assess the possibilities of the system for in vivo imaging, the rSPECT was tested in several common imaging protocols.

a) The first set of experiments was based on ${ }^{99 m}$ Tc-MDP tracer. Whole-body bone scans of a 154-g rat (5 axial positions) and of a $24-\mathrm{g}$ mouse (4 axial positions) were acquired with parallel-hole and pinhole collimators, respectively. Acquisitions were performed after displacement of the bed and ensuring a suitable overlap between two successive displacements. The rat was injected with $6.3 \mathrm{mCi}$ and the mouse with $5.2 \mathrm{mCi}$ of the aforementioned radiopharmaceutical. After two hours uptake, 60 projections were acquired over $360^{\circ}$ in each axial position (ROR: 37.2 $\mathrm{mm}$ and $32.1 \mathrm{~mm}$ for rat and mouse, respectively). The total scan time was two hours in both cases.

b) For the second experiment, a 1-hour heart perfusion scan was carried out using a 128 -g rat and the pinhole collimators. The animal was injected with $3.8 \mathrm{mCi}$ of ${ }^{99 m}$ Tc-MIBI. After one hour uptake, 120 projections were acquired over $360^{\circ}$ (ROR: $31.3 \mathrm{~mm}$ ).

c) Finally, a brain perfusion study was carried out using two Wistar rats and the pinhole collimators. Before acquisition, brain-ischemia was surgically induced in one of the animals. Eighteen hours after intervention both animals were injected with $4 \mathrm{mCi}$ of $99 \mathrm{~m}$ Tc-HMPAO; after one hour uptake, 120 projections of 30 seconds were acquired over $360^{\circ}$ (ROR: $30.1 \mathrm{~mm}$ ).

All the animal experiments were carried out according to the guidelines defined in the European directive 86/609/EEC on the protection of animals used for experimental and other medical purposes. The animals were anesthetized with isoflurane during the scans.

\section{RESULTS}

\section{A. Performance Evaluation}

The performance of the implemented gamma cameras was characterized in terms of spatial resolution and sensitivity for parallel-hole and pinhole collimators $(0.75-\mathrm{mm}$ aperture $)$. Integral uniformity and energy resolution were evaluated without collimators.

1) Spatial Resolution: The results of the planar resolution measurements as a function of source-to-collimator distance are shown in Fig. 4. Theoretical spatial resolution was calculated in both cases assuming a 1.6-mm intrinsic detector resolution. Differences in spatial resolution between both detectors were negligible. Planar spatial resolution for parallel-hole collimators ranged from $2.4 \mathrm{~mm}$ when the source is at $10 \mathrm{~mm}$ to 4.1 $\mathrm{mm}$ when the source-to-collimator distance is $45 \mathrm{~mm}$. With the pinhole collimators, the spatial resolution ranged from $1.2 \mathrm{~mm}$ to $2.4 \mathrm{~mm}$ at the same distances.

2) Sensitivity: Fig. 5 shows the camera sensitivity with parallel-hole and pinhole collimators $(0.75-\mathrm{mm}$ aperture). These values are in good agreement with the expected theoretical values (calculated as the geometric efficiency of the collimators). The sensitivity of pinhole collimator equipped with bigger apertures (not yet available) is expected to be proportional to that shown in Fig. 5, with the proportionality factor being the 

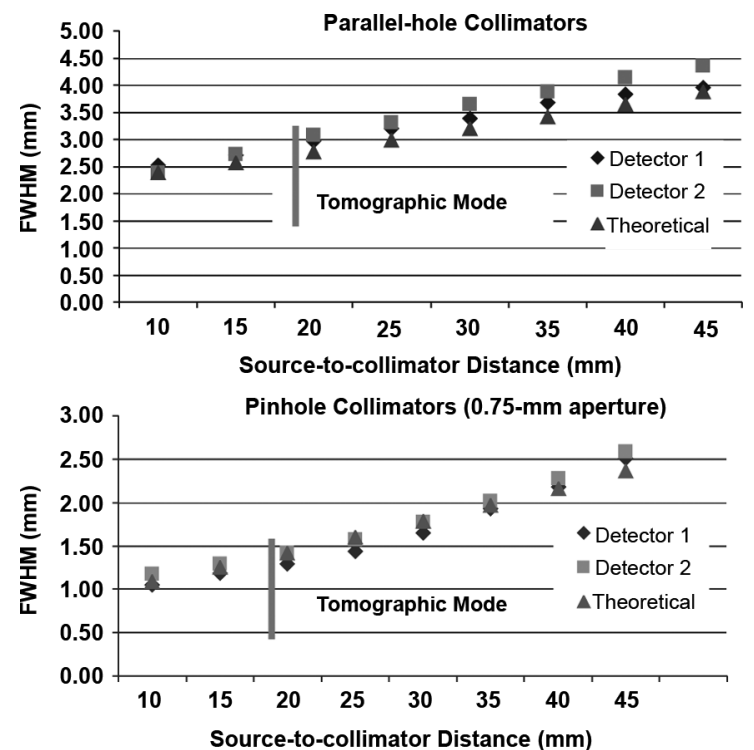

Fig. 4. Planar spatial resolution (FWHM) as a function of the selected source-to-collimator distance. Measurements were made using the parallel-hole collimators (top) and the pinhole collimators (0.75-mm aperture) (bottom). In both cases, a 20\% symmetric energy window was used. The vertical line of both plots indicates the minimum usable ROR for tomographic studies $(\sim 20 \mathrm{~mm})$.

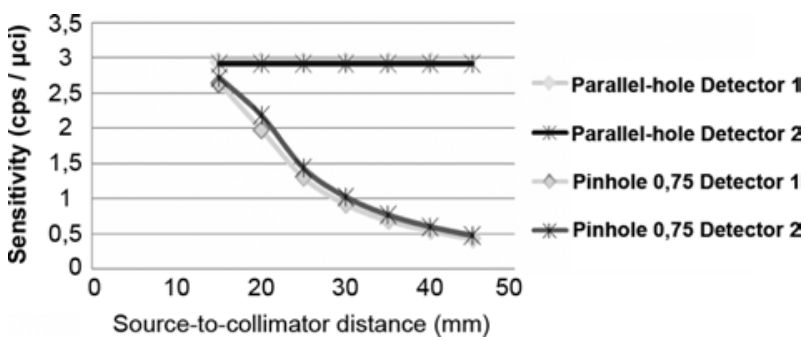

Fig. 5. Sensitivity of the cameras as a function of the selected source-to-collimator distance. Measurements were made using the parallel-hole collimators and the pinhole collimators ( $0.75-\mathrm{mm}$ aperture). In both cases a $20 \%$ symmetric energy window was used.

TABLE I

INTEGRAL UNIFORMITY (\%) AND ENERGY RESOLUTION (\%) AT 140 KEV

\begin{tabular}{lcc}
\hline \hline & Camera 1 & Camera 2 \\
\hline $\begin{array}{c}\text { Uncorrected Intrinsic Non- } \\
\quad \text { Uniformity (UFOV) }\end{array}$ & $12.9 \%$ & $10.4 \%$ \\
$\begin{array}{c}\text { Uncorrected Intrinsic Non- } \\
\text { Uniformity (CFOV) }\end{array}$ & $8.6 \%$ & $7.9 \%$ \\
$\begin{array}{c}\text { Energy Resolution } \\
\text { (UFOV, Average) }\end{array}$ & $10.3 \%$ & $11.1 \%$ \\
$\quad \begin{array}{l}\text { Energy Resolution } \\
\text { (UFOV, Best Crystal) } \\
\text { Energy Resolution }\end{array}$ & $8.2 \%$ & $9.3 \%$ \\
(UFOV, Worst Crystal) & $19.2 \%$ & $18.6 \%$ \\
\hline \hline
\end{tabular}

ratio of the square of the aperture diameters. Sensitivity shown in the figure for pinhole collimator was obtained at the central axis of the pinhole; a $12.8 \%$ drop in sensitivity was measured $1 \mathrm{~cm}$ off axis which also agrees with the expected theoretical values. The energy resolution at various locations and the integral uniformity over the CFOV and UFOV are presented in Table I.

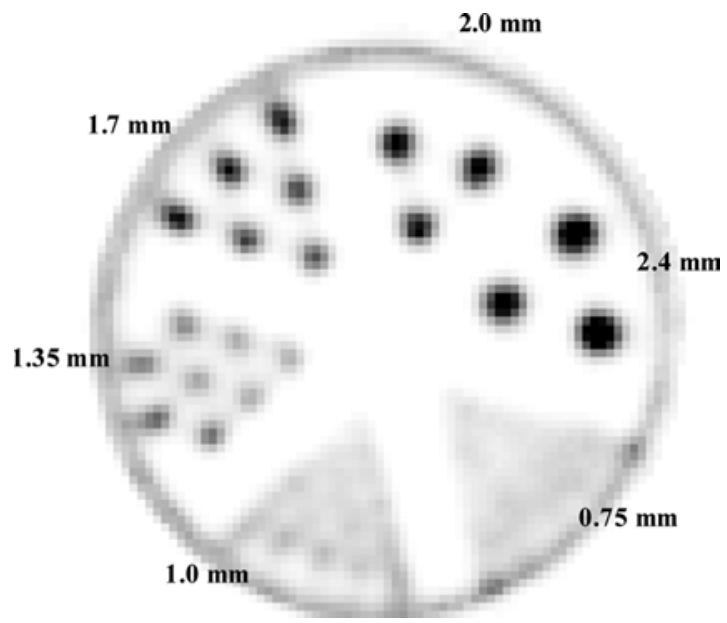

Fig. 6. Axial slice of the micro-Derenzo phantom. The image was acquired in 120 minutes using the pinhole collimator. Hot rods of $1.35 \mathrm{~mm}$ are clearly resolved in the image.

Average energy resolution (both cameras) is $10.7 \%$ over the UFOV. Integral uniformity is less than $2.0 \%$ in both gamma cameras after uniformity correction.

\section{B. SPECT Phantom Imaging}

A transverse slice through the reconstructed volume is shown in Fig. 6, where the largest four sectors are clearly resolved. The image was reconstructed using 3D OSEM with 16 iterations and 8 subsets (voxel size $0.4 \mathrm{~mm} \times 0.4 \mathrm{~mm} \times 0.8 \mathrm{~mm}$ ).

\section{In-Vivo Imaging}

1) ${ }^{99 \mathrm{~m}} \mathrm{Tc}-\mathrm{MDP}$ Scans: Figs. 7(a) and 7(c) show maximum intensity projection images (MIP) of two bone studies (mouse and rat, respectively) obtained with ${ }^{99 \mathrm{~m}} \mathrm{Tc}-\mathrm{MDP}$ tracer. The mouse image was acquired with the pinhole collimators and reconstructed using 3D OSEM with 16 iterations and 8 subsets (pixel size: $0.4 \mathrm{~mm}$; slice thickness: $0.8 \mathrm{~mm}$ ). The rat image was acquired with parallel-hole collimators and reconstructed using 2D FBP with a ramp filter (top) and 2D OSEM with 8 iterations and 10 subsets (bottom). In both cases the pixel size was $0.4 \mathrm{~mm}$ and the slice thickness $0.8 \mathrm{~mm}$. Fig. 7(c) also shows different axial sections of the rat study for both reconstructions. We can observe the higher resolution achieved using the iterative algorithm with respect to the filtered backprojection.

2) ${ }^{99 m}$ Tc-MIBI Scan: Fig. 7(b)-top shows standard views of a heart perfusion scan. Data were reconstructed using the FDK algorithm (pixel size: $1.11 \mathrm{~mm}$; slice thickness: 1.11 $\mathrm{mm}$ ) with a Butterworth filter (cutoff: 0.35/pixel; order: 12). Fig. 7(b)-bottom shows the same study reconstructed using 3D OSEM with 5 iterations and 8 subsets (pixel size: $0.4 \mathrm{~mm}$; slice thickness: $0.8 \mathrm{~mm}$ ). We can also observe in this image the higher resolution achieved using the iterative algorithm with respect to the FDK algorithm.

3) ${ }^{99 \mathrm{~m}} \mathrm{Tc}-H M P A O$ Scans: Fig. 7(d)-top shows axial slices of a brain perfusion scan performed using the control specimen. The image obtained from the animal with the surgically induced ischemia is shown in Fig. 7(d)-bottom. The perfusion lesion is clearly visible in this animal (see the arrow in Fig. 7(d)-bottom). 


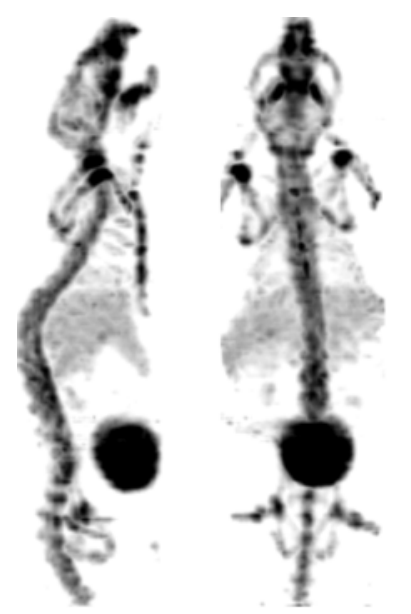

(a)

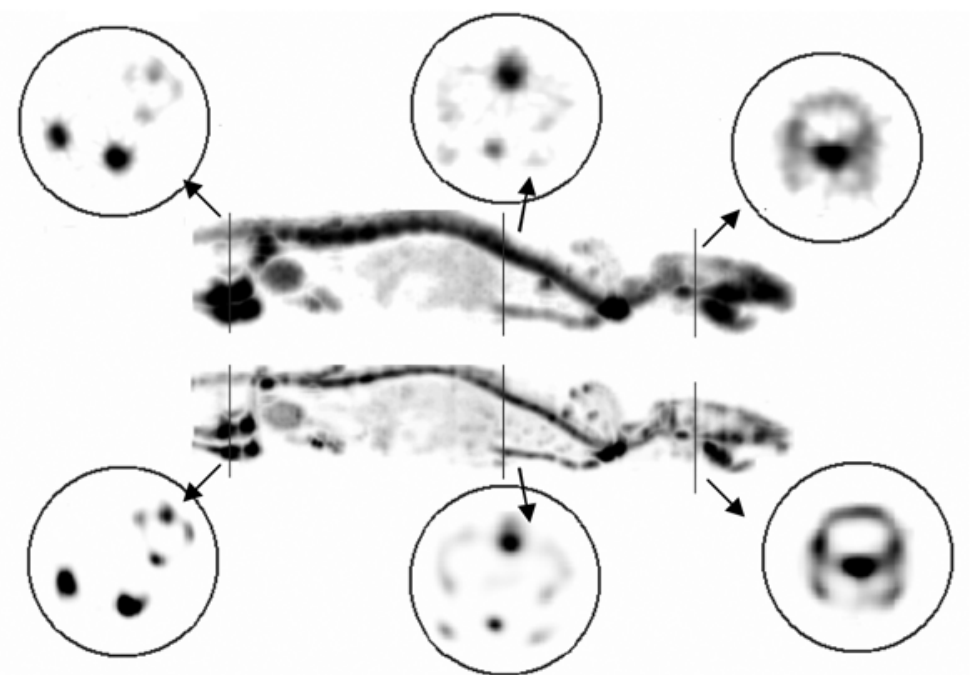

(c)
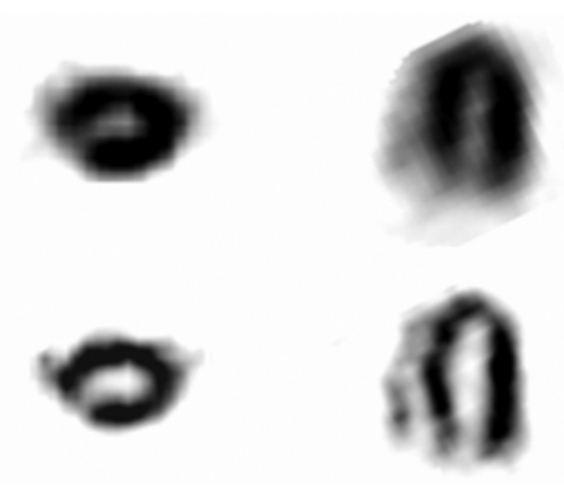

(b)
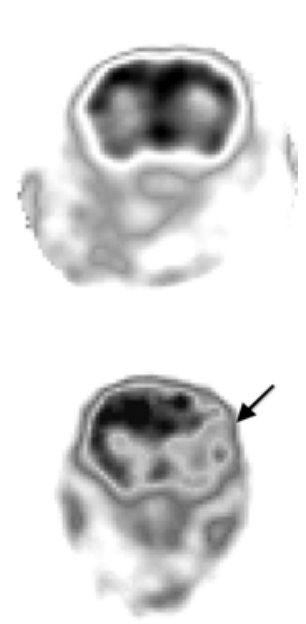

(d)

Fig. 7. (a) SPECT image MIP render of a $24-\mathrm{g}$ mouse bone scan ( $\left.{ }^{99 \mathrm{~m}} \mathrm{Tc}-\mathrm{MDP}\right)$. Image was acquired using pinhole collimators, data were reconstructed using $3 \mathrm{D}$ OSEM. (b) From left to right: SA (short axis), VLA (vertical long axis) and HLA (horizontal long axis) views of a 128-g rat heart perfusion scan ( ${ }^{99 m}$ Tc-MIBI). Data were acquired using pinhole collimators and reconstructed using FDK (top) and with 3D OSEM (bottom). (c) SPECT image MIP render of a 154-g rat bone scan $\left({ }^{99 \mathrm{~m}} \mathrm{Tc}-\mathrm{MDP}\right)$. Image was acquired using parallel-hole collimators and reconstructed using 2D FBP (top) and 2D OSEM (bottom). The image also shows different axial sections for both reconstructions (the position of these sections in the volume is indicated with vertical lines). (d) Axial slice of a brain perfusion scan ( ${ }^{99 \mathrm{~m}} \mathrm{Tc}$-HMPAO) carried out using a control rat (top) and a rat with a surgically induced brain-ischemia (bottom). Data were acquired using pinhole collimators; images were reconstructed using 3D OSEM.

In both cases, data were reconstructed using the 3D OSEM algorithm with 2 iterations and 8 subsets (pixel size: $0.4 \mathrm{~mm}$; slice thickness: $0.8 \mathrm{~mm}$ ).

\section{DISCUSSION}

The main interest of this work was to evaluate the potential of using an approach based on small-area gamma cameras for the implementation of a small-animal tomographic system. Although some commercial systems such as the Gamma Medica-Ideas X-SPECT [33], are also based on a configurable number (1-4) of gamma cameras with relatively small-area (125 $\mathrm{mm} \times 125 \mathrm{~mm}$ active area per camera), the proposed 4-head system uses roughly $50 \%$ less detector material than an $\mathrm{X}$-SPECT equipped with a single camera, thus considerably reducing costs, while providing acceptable performance for a great variety of common protocols.
Despite the fact that the rSPECT prototype is functional and the imaging performance indicators are consistent with expectations, there is still some room for improvement. Regarding parallel-hole configuration, a matched collimator designed to permit the lead septa to be located in the dead regions between detector elements can improve the sensitivity of the detectors [34]. Regarding pinhole configuration, optimization of the iterative reconstruction algorithms with pinhole collimator will be addressed in future studies in order to include modeling of collimator, septal penetration [35], [36], and depth-of-interaction in the detector array as a function of the angle-of-incidence on the detectors.

\section{SUMmary AND CONCluSiONS}

We have designed and developed a versatile and modular gamma camera system based on small-field-of-view and highspatial-resolution detectors. The performance of the resulting 
cameras was evaluated in terms of spatial resolution, energy resolution, sensitivity and uniformity, showing good characteristics for the use of this technology in clinical applications similar to that reported in [20], [22], [37]. The possibilities of these detectors for small-animal SPECT applications were evaluated by implementing a dedicated tomograph. Pinhole and parallel-hole collimators were used to acquire SPECT images of mice and rats and studies were reconstructed with analytical and iterative algorithms.

Tomographic spatial resolution and reconstructed image quality were greatly improved using iterative algorithms to compensate the lack of magnification of pinhole collimators and the poor resolution of parallel-hole collimators when applied to small-animals. Imaging parameters such as injected dose or imaging time were not optimized for the system configuration, but yielded reasonable imaging times for common acquisition protocols. Although the rSPECT prototype was designed for cost-effective routine small-animal imaging, studies of living mice and rats obtained with this system demonstrate its ability to provide high-resolution images for in vivo research.

\section{ACKNOWLEDGMENT}

The authors thanks SEDECAL Engineering Department for their support during the system development.

\section{REFERENCES}

[1] R. J. Jaszczak, L. Jian Ying, W. Huili, M. R. Zalutsky, and R. E. Coleman, "Pinhole colimation for ultrahigh-resolution small-field-of-view SPECT," Phys. Med. Biol., vol. 39, pp. 425-437, 1994.

[2] I. Koichi, M. Takao, Y. Yoshiharu, P. Marco, F. Toru, M. Yasuhiro, N. Sadahiko, T. Nagara, S. Hiroshi, and K. Junji, "Ultra-high resolution SPECT system using four pinhole collimators for small animal studies," J. Nucl. Med., vol. 36, pp. 2282-2287, 1995.

[3] K. Ogawa, T. Kawade, K. Nakamura, A. Kubo, and T. Ichihara, "Ultrahigh resolution pinhole SPECT for small animal study," IEEE Trans. Nucl. Sci., vol. 45, pp. 3122-3126, 1998.

[4] L. R. Furenlid, D. W. Wilson, Y. Chen, H. Kim, P. J. Pietraski, M. J. Crawford, and H. H. Barrett, "FastSPECT II: A second-generation high-resolution dynamic SPECT imager," IEEE Trans. Nucl. Sci., vol. 51, pp. 631-635, 2004.

[5] K. Inoue, K. Tani, I. O. Umeda, M. Yamaguchi, Y. Mutou, M. Fukushi, and H. Fujii, "Evaluation of SPECT/CT performance for optimal small animal imaging," in Proc. World Molecular Imaging Congress, Nice, France, 2008.

[6] F. Beekman and B. Vastenhouw, "Submillimeter total-body murine imaging with U-SPECT-I," J. Nucl. Med., vol. 48, pp. 487-493, 2007.

[7] F. van der Have, B. Vastenhouw, R. M. Ramakers, W. Branderhorst, J. O. Krah, C. Ji, S. G. Staelens, and F. J. Beekman, "U-SPECT-II: An ultra-high-resolution device for molecular small-animal imaging," J. Nucl. Med., vol. 50, pp. 599-605, Apr. 2009.

[8] H. Kim, L. R. Furenlid, M. J. Crawford, D. W. Wilson, H. Barber, T. E. Peterson, W. J. C. Hunter, Z. Liu, J. M. Wolfenden, and H. H. Barrett, "SemiSPECT: A small-animal single-photon emission computed tomography (SPECT) imager based on eight cadmiun zinc telluride (CZT) detector arrays," Med. Phys., vol. 33, pp. 465-473, 2006.

[9] N. U. Schramm, G. Ebel, U. Engeland, T. Schurrat, M. Béhé, and T. M. Behr, "High-resolution SPECT using multipinhole collimation," IEEE Trans. Nucl. Sci., vol. 50, pp. 315-320, 2003.

[10] D. W. Austin, M. J. Paulus, S. S. Gleason, R. A. Mintzer, S. A. Siegel, S. D. Figueroa, T. J. Hoffman, and J. S. Wall, "Design and performance of a new SPECT detector for multimodality small animal imaging platforms," in Proc. IEEE Nuclear Science Symp. and Medical Imaging Conf. Rec., 2006, pp. 3008-3011.
[11] L. R. MacDonald, B. E. Patt, J. S. Iwanczyk, B. M. W. Tsui, Y. Wang, E. C. Frey, D. E. Wessell, P. D. Acton, and H. F. Kung, "Pinhole SPECT of mice using the LumaGEM gamma camera," IEEE Trans. Nucl. Sci., vol. 48, pp. 830-836, 2001.

[12] D. P. McElroy, L. R. MacDonald, F. J. Beekman, Y. Wang, B. E. Patt, J. S. Iwanczyk, B. M. W. Tsui, and E. J. Hoffman, "Performance evaluation of a-SPECT: A high resolution desktop pinhole SPECT system for imaging small animals," IEEE Trans. Nucl. Sci., vol. 49, pp. 2139-2147, 2002.

[13] T. Y. Song, Y. Choi, J. H. Jung, B. J. Min, K. J. Hong, Y. S. Choe, K. Lee, and B. Kim, "Performance amelioration for small animal SPECT using optimized pinhole collimator and image correction technique," IEEE Trans. Nucl. Sci., vol. 52, pp. 1396-1400, 2005.

[14] A. D. Guerra, A. Bartoli, N. Belcari, D. Herbert, A. Motta, A. Vaiano, G. D. Domenico, N. Sabba, E. Moretti, G. Zavattini, M. Lazzarotti, L. Sensi, M. Larobina, and L. Uccelli, "Performance evaluation of the fully engineered YAP-(S)PET scanner for small animal imaging," IEEE Trans. Nucl. Sci., vol. 53, pp. 1078-1083, 2006.

[15] A. G. Weisenberger, R. Wojcik, E. L. Bradley, P. Brewer, S. Majewski, J. Qian, A. Ranck, M. S. Saha, K. Smith, M. F. Smith, and R. E. Welsh, "SPECT-CT system for small animal imaging," IEEE Trans. Nucl. Sci., vol. 50, pp. 74-79, 2003.

[16] G. Loudos, S. Majewski, R. Wojcik, A. G. Weisenberger, N. Sakellios, K. Nikita, N. Uzunoglu, P. Bouziotis, S. Xanthopoulos, and A. Varvarigou, "Performance evaluation of a dedicated camera suitable for dynamic radiopharmaceuticals evaluation in small animals," IEEE Trans. Nucl. Sci., vol. 54, pp. 454-460, 2007.

[17] E. L. Bradley, J. Cella, S. Majewski, V. Popov, J. Qian, M. S. Saha, M. F. Smith, A. G. Weisenberger, and R. E. Welsh, "A compact gamma camera for biological imaging," IEEE Trans. Nucl. Sci., vol. 53, pp. 59-65, 2006.

[18] S. R. Meikle, P. Kench, A. G. Weisenberger, R. Wojcik, M. F. Smith, S. Majewski, S. Eberl, R. R. Fulton, A. B. Rosenfeld, and M. J. Fulham, "A prototype coded aperture detector for small animal SPECT," IEEE Trans. Nucl. Sci., vol. 49, pp. 2167-2171, 2002.

[19] S. Siegel, J. J. Vaquero, L. Aloj, J. Seidel, E. M. Jagoda, W. Gandler, W. Eckelman, and M. Green, "Initial results from a PET/planar small animal imaging system," IEEE Trans. Nucl. Sci., vol. 46, pp. 571-576, 1999.

[20] P. D. Olcott, F. Habte, A. M. Foudray, and C. S. Levin, "Performance characterization of a miniature, high sensitivity gamma ray camera," IEEE Trans. Nucl. Sci., vol. 54, pp. 1492-1497, 2007.

[21] M. J. Moré, P. J. Goodale, S. Majewski, and M. B. Williams, "Evaluation of gamma cameras for use in dedicated breast imaging," IEEE Trans. Nucl. Sci., vol. 53, pp. 2675-2679, 2006.

[22] M. P. Tornai, C. N. Brzymialkiewicz, M. L. Bradshaw, J. E. Bowsher, B. E. Patt, J. S. Iwanczyk, J. Li, and L. R. MacDonald, "Comparison of compact gamma cameras with 1.3- and 2.0-mm quantized elements for dedicated emission mammotomography," IEEE Trans. Nucl. Sci., vol. 52, pp. 1251-1256, 2005.

[23] E. Lage, J. J. Vaquero, A. Sisniega, S. Espana, G. Tapias, M. A. A. Rodriguez-Ruano, J. E. Ortuno, A. Udias, and M. Desco, "Design and performance evaluation of a coplanar multimodality scanner for rodent imaging," Phys. Med. Biol., vol. 54, pp. 5427-5441, 2009.

[24] P. D. Olcott, J. A. Talcott, C. S. Levin, F. Habte, and A. M. K. Foudray, "Compact readout electronics for position sensitive photomultiplier tubes," IEEE Trans. Nucl. Sci., vol. 52, pp. 21-27, 2005.

[25] V. Popov, S. Majewski, and A. G. Weisenberger, "Readout electronics for multianode photomultiplier tubes with pad matrix anode layout," in Proc. IEEE Nuclear Science Symp. and Medical Imaging Conf. Rec., 2003, vol. 3, pp. 2156-2159.

[26] J. J. Vaquero, E. Lage, L. Ricon, M. Abella, E. Vicente, and M. Desco, "RPET detectors design and data processing," in Proc. IEEE Nuclear Science Symp. and Medical Imaging Conf. Rec., 2005, vol. 5, pp. 2885-2889.

[27] J. Pascau, J. J. Vaquero, M. Abella, R. Cacho, E. Lage, and M. Desco, "Multimodality workstation for small animal image visualization and analysis," Mol. Imag. Biol., vol. 8, pp. 97-98, 2006.

[28] D. Beque, J. Nuyts, G. Bormans, P. Suetens, and P. Dupont, "Characterization of pinhole SPECT acquisition geometry," IEEE Trans. Med. Imag., vol. 22, pp. 599-612, 2003.

[29] L. A. Feldkamp, L. C. Davis, and J. W. Kress, "Practical cone beam algorithm," J. Opt. Soc. Amer., vol. 1, pp. 612-619, 1984. 
[30] H. M. Hudson and R. S. Larkin, "Accelerated image reconstruction using ordered subsets of projection data," IEEE Trans. Med. Imag., vol. 13, pp. 601-609, 1994.

[31] D. Pareto, A. Cot, J. Pavia, C. Falcon, I. Juvells, F. Lomena, and D. Ros, "Iterative reconstruction with compensation of the spatial variant fan beam collimator response in neurotransmission SPET imaging," Eur. J. Nucl. Med. Mol. Imag., vol. 30, pp. 1322-1329, 2003.

[32] A. Cot, C. Falcon, C. Crespo, C. J. Sempau, D. Pareto, S. Bullich, F. Lomena, F. Calvino, J. Pavia, and D. Ros, "Absolute quantification in dopaminergic neurotransmission SPECT using a monte carlo-based scatter correction and fully 3-dimensional reconstruction," $J$. Nucl. Med., vol. 46, pp. 1497-1504, 2005.

[33] Gamma Medica-Ideas [Online]. Available: http://www.gm-ideas.com

[34] W. Xia, J. Seidel, J. W. Kakarekad, T. J. Pohidad, D. E. Milenice, J. Proffittf, S. Majewski, A. G. Weisenberger, M. V. Green, and P. L. Choykeb, "MONICA: a compact, portable dual gamma camera system for mouse whole-body imaging," Nucl. Med. Biol., vol. 1, pp. 245-253, 2010.
[35] R. Accorsi and S. D. Metzler, "Analytic determination of the resolution-equivalent effective diameter of a pinhole collimator," IEEE Trans. Med. Imag., vol. 23, pp. 750-763, 2004.

[36] S. D. Metzler and R. Accorsi, "Resolution- versus sensitivity-effective diameter in pinhole collimation: Experimental verification," Phys. Med. Biol., vol. 50, pp. 5005-5017, 2005.

[37] F. Sánchez, J. M. Benlloch, B. Escat, N. Pavón, E. Porras, D. KadiHanifi, J. A. Ruiz, F. J. Mora, and A. Sebastia, "Design and tests of a portable mini gamma camera," Med. Phys., vol. 31, pp. 1384-1397, 2004. 